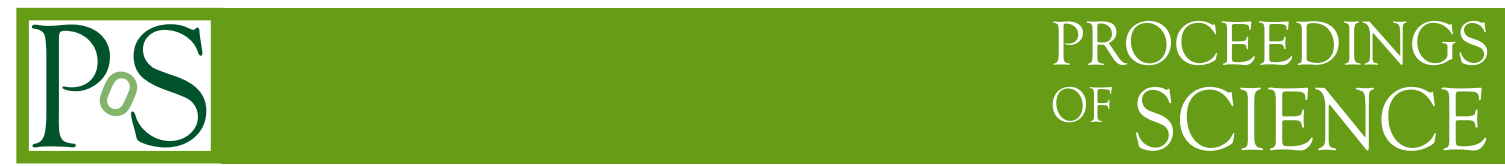

\title{
Future applications of the Yang-Mills gradient flow in lattice QCD
}

\section{Lüscher*}

CERN, Physics Department, 1211 Geneva 23, Switzerland

E-mail: luscheremail.cern.ch

The Yang-Mills gradient flow has many interesting applications in lattice QCD. In this talk, some recent and possible future uses of the flow are discussed, emphasizing the underlying theoretical concepts rather than any computational aspects.

31st International Symposium on Lattice Field Theory - LATTICE 2013

July 29 - August 3, 2013

Mainz, Germany

\footnotetext{
* Speaker.
} 


\section{Introduction}

In the past few years, the Yang-Mills gradient flow turned out to be a theoretically attractive and powerful tool for non-perturbative studies of QCD. A key feature of the flow is certainly the fact that local fields constructed at positive flow time renormalize in a simple way, however complicated they may be [1]-3]. Correlation functions of such fields calculated in lattice QCD therefore have a well-defined continuum limit and thus provide interesting probes of the universal properties of the theory.

Apart from a brief recap of some basic facts and equations, the subject will not be reviewed in this talk. Instead the focus is on applications of the gradient flow which have not or just barely been considered so far. The computation of the chiral condensate via the quark density at positive flow times is one of them, and its discussion here partly serves as an introduction to important concepts such as the small flow-time expansion of local fields. There are potentially very interesting uses of the latter in studies of QCD at non-zero temperatures and in computations of electro-weak transition matrix elements, for example.

Another topic covered is the application of the gradient flow in the context of renormalization and the continuum limit. While the flow itself is not a renormalization group transformation, non-perturbative renormalization can be based on observables at positive flow times. Calculations of the scale evolution of couplings and fields, using step scaling, as well as systematic constructions of coarse-grid actions through a matching procedure are likely to profit from the use of such observables, since their expectation values can often be accurately computed with a modest effort.

\section{The Yang-Mills gradient flow in QCD}

\subsection{Flow equations}

The Yang-Mills gradient flow $B_{\mu}(t, x), t \geq 0$, of $\mathrm{SU}(3)$ gauge potentials is defined by the flow equation

$$
\begin{aligned}
& \partial_{t} B_{\mu}=D_{v} G_{v \mu}, \\
& G_{\mu v}=\partial_{\mu} B_{v}-\partial_{v} B_{\mu}+\left[B_{\mu}, B_{v}\right], \quad D_{\mu}=\partial_{\mu}+\left[B_{\mu}, \cdot\right],
\end{aligned}
$$

and the initial condition $B_{\mu}(0, x)=A_{\mu}(x)$, where $A_{\mu}(x)$ denotes the fundamental gauge field integrated over in the QCD functional integral. Since the flow equation is of first order in the derivatives with respect to the flow time $t$, the gauge potentials $B_{\mu}(t, x)$ are uniquely determined by their initial value at $t=0$ and are thus well-defined functions of the fundamental gauge field.

Starting from the fundamental quark field $\chi(0, x)=\psi(x)$ at flow time zero, an associated flow $\chi(t, x)$ of quark fields may be similarly defined through the equations

$$
\begin{aligned}
& \partial_{t} \chi=\Delta \chi, \\
& \Delta=D_{\mu} D_{\mu}, \quad D_{\mu}=\partial_{\mu}+B_{\mu} .
\end{aligned}
$$

Clearly, $\chi(t, x)$ is a uniquely determined linear function of the fundamental quark field and a nonlinear function of the gauge potential $B(s, y), 0 \leq s \leq t$, and therefore of the fundamental gauge 
field. In the flow equation (2.3), the gauge-covariant Laplacian $\Delta$ could be replaced by the square of the Dirac operator, but while this choice would be mathematically appealing, it does not appear to offer any particular advantages in the present context.

It goes without saying that a regularization is required in the quantized theory for the gradient flow and the associated quark flow to be well defined. Dimensional regularization is a possible choice in perturbation theory and it is straightforward to set up both the gauge and the quark flow on the lattice [1. 3]. For simplicity, I however continue to use a continuum notation and implicitly assume a lattice regularization.

\subsection{Smoothing property}

In the direction of increasing flow time, the evolution of the time-dependent gauge and quark fields tends to have a smoothing effect. It is possible to show this by expanding the solution of the flow equations in powers of the fundamental fields. At the leading order of the expansion,

$$
\begin{aligned}
& B_{\mu}(t, x)=\int \mathrm{d}^{4} y K_{t}(x-y) A_{\mu}(y)+\text { gauge \& non-linear terms, } \\
& \chi(t, x)=\int \mathrm{d}^{4} y K_{t}(x-y) \psi(y)+\ldots, \quad K_{t}(z) \propto \exp \left\{-\frac{z^{2}}{4 t}\right\},
\end{aligned}
$$

the fields are then seen to be smoothed with a Gaussian kernel over a spherical range of distances with radius roughly equal to $\sqrt{8 t}$. The smoothing property is unchanged at higher orders and is also observed in numerical studies, the smoothing range being practically the same as the one given by the leading-order formulae (2.5), (2.6).

The Yang-Mills gradient flow was probably first considered in mathematics by Atiyah and Bott [ [ 4 in their seminal work on the Morse theory of the space of gauge fields on two-dimensional manifolds. In lattice QCD, field smoothing techniques are in use since many years. Repeated "stout link smearing" [5] in fact amounts to a numerical integration of the flow equation (2.1) using an Euler scheme, while the source-smearing operations proposed in refs. [6, 7] solve the quark flow equation (2.3) on an equal-time hyperplane, albeit without simultaneously evolving the gauge field.

\subsection{Observables}

Gauge-invariant composite fields constructed at positive flow time $t$, such as

$$
\begin{aligned}
& E_{t}=-\frac{1}{2} \operatorname{tr}\left\{G_{\mu \nu} G_{\mu \nu}\right\} \\
& P_{t}^{r s}=\bar{\chi}_{r} \gamma_{5} \chi_{s}, \quad S_{t}^{r s}=\bar{\chi}_{r} \chi_{s}, \quad r, s: \text { flavour labels },
\end{aligned}
$$

are well-defined functions of the fundamental field variables and are therefore possible observables like the ordinary composite fields. In particular, one may be interested in their correlation functions and, more generally, the QCD expectation values of any product of local fields at zero and non-zero flow times. Clearly, an observable $\mathcal{O}_{t}(x)$ that is locally constructed at some flow time $t>0$ is not a local expressions in the fundamental fields, but essentially only depends on the field variables in a spherical region of space-time centered at $x$, which I will refer to as the footprint of the observable (see fig. 1). 


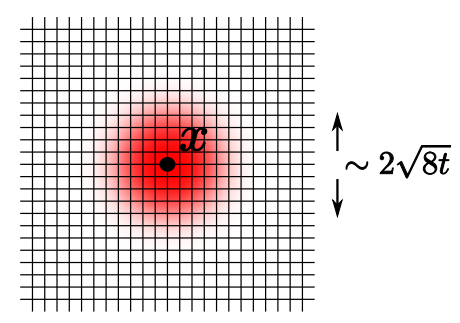

Figure 1: Local fields $\mathcal{O}_{t}(x)$ constructed at flow time $t>0$ depend on the fundamental field variables in a region of space-time approximately $2 \sqrt{8 t}$ wide (red area). Further away from the point $x$, the sensitivity to the basic fields decreases like a Gaussian and very rapidly becomes totally negligible.

The smoothing property of the gradient flow and the associated quark flow implies that correlation functions of fields at non-zero flow times have no short-distance singularities. Renormalization is nevertheless required, but turns out to be extremely simple. Explicitly, if $\mathcal{O}_{t}(x)$ is a bare, gaugeinvariant composite field at flow time $t>0$ of degree $n$ and $\bar{n}$ in the quark and antiquark fields, the renormalized field is given by

$$
\mathcal{O}_{\mathrm{R}, t}=\left(Z_{\chi}\right)^{\frac{1}{2}(n+\bar{n})} \mathcal{O}_{t},
$$

where the renormalization constant $Z_{\chi}$ is independent of $t$. In particular, the field (2.7) does not require renormalization and the chiral densities (2.8) renormalize with the same factor $Z_{\chi}$.

The proof of these statements [2, 3] is based on an exact representation of the correlation functions through a local field theory in $4+1$ dimensions, the extra dimension being the flow time. Zinn-Justin and Zwanziger [8] introduced the representation many years ago in their work on the renormalization of the Langevin equation. In the pure gauge theory, the latter actually coincides with the flow equation (2.1) except for the fact that it includes a noise term, which complicates the situation and requires a renormalization of the Langevin time, for example.

\section{Chiral condensate}

In lattice QCD, the expectation value of the scalar density $\bar{u} u+\bar{d} d$ of the up and down quarks diverges like the second or third inverse power of the lattice spacing when the continuum limit is taken. The divergent terms are proportional to the light-quark masses if the lattice theory preserves chiral symmetry, but also in these cases their subtraction tends to give rise to important significance losses or even some conceptual issues. Using the gradient flow, this problem can now be elegantly bypassed [纤].

\subsection{Flow-time dependent condensate}

Since the flow equations are chirally invariant, the quark field at non-zero flow times, $\chi(t, x)$, transforms in the same way as the fundamental field $\psi(x)$ under global chiral rotations. In particular, the light-quark chiral densities

$$
S_{t}^{r s} \pm P_{t}^{r s}, \quad r, s \in\{u, d\},
$$




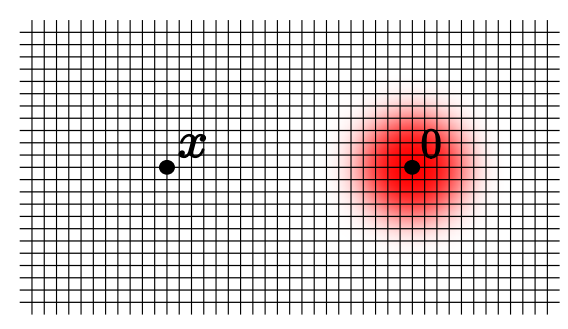

Figure 2: As long as $t$ is strictly positive, the pseudo-scalar two-point function in the PCAC relation (3.3) has no short-distance singularities. Moreover, at distances $|x| \gg \sqrt{8 t}$, the transfer matrix formalism may be invoked to show that the correlation function falls off proportionally to $\mathrm{e}^{-M_{\pi}|x|}$.

transform according to the $\left(\frac{1}{2}, \frac{1}{2}\right)$ representation of the $\mathrm{SU}(2)_{\mathrm{L}} \times \mathrm{SU}(2)_{\mathrm{R}}$ chiral symmetry group that acts on the up and down quark fields. The "time-dependent condensate"

$$
\Sigma_{t}=-\frac{1}{2}\left\langle S_{t}^{u u}+S_{t}^{d d}\right\rangle
$$

is therefore an order parameter for the spontaneous breaking of this symmetry.

From a technical point of view, the condensate (3.2) is an attractive quantity, because no powerdivergent terms need to be subtracted, when the continuum limit is taken, and only multiplicative renormalization is required according to eq. (2.9). Moreover, calculations of $\Sigma_{t}$ through numerical simulation are straightforward and quickly yield accurate results.

\subsection{Relation to $\Sigma$}

The time-dependent condensate $\Sigma_{t}$ can be directly used for studies of spontaneous chiral symmetry breaking at non-zero temperatures, for example, but one may nevertheless be interested in its relation to the standard chiral condensate $\Sigma$.

The link between the two condensates is provided by the PCAC relation. When probed by the pseudo-scalar density $P_{t}^{d u}$ at flow time $t$ and integrated over space-time, the relation implies

$$
\Sigma_{t}=-\frac{M_{\pi}^{2} F_{\pi}}{2 G_{\pi}} \int \mathrm{d}^{4} x\left\langle P^{u d}(x) P_{t}^{d u}(0)\right\rangle,
$$

where $M_{\pi}, F_{\pi}$ and $G_{\pi}$ denote the pion mass, the pion decay constant and the vacuum-to-pion matrix element of the density $P^{u d}$ (see fig. 2). At large distances, the two-point function in this equation falls off exponentially and another constant, $G_{\pi, t}$, may be defined through the asymptotic formula

$$
\int \mathrm{d}^{3} \vec{x}\left\langle P^{u d}(x) P_{t}^{d u}(0)\right\rangle \underset{x_{0} \rightarrow \infty}{\sim}-\frac{G_{\pi} G_{\pi, t}}{M_{\pi}} \mathrm{e}^{-M_{\pi} x_{0}} .
$$

Now when the up and down quark masses $m_{u}, m_{d}$ are sent to zero, the integral in eq. (3.3) diverges at large $x$ and a few lines of algebra then show that

$$
\Sigma=\lim _{m_{u}=m_{d} \rightarrow 0} \Sigma_{t} \frac{G_{\pi}}{G_{\pi, t}} .
$$

In the $\mathrm{SU}(2)$ chiral limit, the time-dependent chiral condensate $\Sigma_{t}$ thus coincides with the standard condensate $\Sigma$ up to a computable proportionality constant. 


\begin{tabular}{ccc}
$\sqrt{8 t}[\mathrm{fm}]$ & $a^{3} Z_{P}^{-1} \Sigma_{t}$ & $a^{3} Z_{P}^{-1} \Sigma_{t} \frac{G_{\pi}}{G_{\pi, t}}$ \\
\hline \hline 0.4 & $0.0006277(95)$ & $0.003962(61)$ \\
0.5 & $0.0004251(58)$ & $0.003872(55)$ \\
0.6 & $0.0002911(36)$ & $0.003785(51)$ \\
0.7 & $0.0002017(23)$ & $0.003711(48)$ \\
\hline \hline
\end{tabular}

Table 1: Simulation results for the unrenormalized time-dependent condensate and the ratio of constants on the right of eq. (3.5). The values are given in lattice units and the quoted errors are statistical only.

\subsection{Chiral perturbation theory}

How exactly the chiral limit (3.5) is reached can be predicted using chiral perturbation theory. The important point to note is that local fields at non-zero flow time with a footprint much smaller than the Compton wave length of the pion are indistinguishable from strictly local fields when only their matrix elements between low-energy pion states are considered. In the effective chiral theory, and if

$$
8 t M_{\pi}^{2} \ll 1,
$$

the time-dependent densities (3.1) are therefore represented by the same fields as the ordinary chiral densities, except for the fact that the coefficients in these expressions are not the same.

To one-loop order of SU(2) chiral perturbation theory [9], the formula

$$
\Sigma_{t} \frac{G_{\pi}}{G_{\pi, t}}=\Sigma\left\{1-\frac{3 M_{\pi}^{2}}{32 \pi^{2} F_{\pi}^{2}} \ln \left(M_{\pi}^{2} / \Lambda_{t}^{2}\right)+\ldots\right\}
$$

is then obtained, where

$$
\bar{l}_{t}=\left.\ln \left(\Lambda_{t}^{2} / M^{2}\right)\right|_{M=139.6 \mathrm{MeV}}
$$

is a new low-energy effective constant. While the form of the expansion (3.7) looks familiar, the constant $\bar{l}_{t}$ is unrelated to the well-known low-energy constants [9] and moreover depends on the flow time $t$.

\subsection{Numerical experiment}

As already mentioned, the time-dependent condensate is easily computed through numerical simulation of the lattice theory. For illustration, the results obtained in a first study of this kind [3] are now briefly recalled. In this run (labeled $I_{1}$ in ref. [11]), the $\mathrm{O}(a)$-improved lattice theory with $2+1$ flavours of Wilson quarks was simulated on a $64 \times 32^{3}$ lattice with open boundary conditions in time [10]. At the chosen point in parameter space, the lattice spacing is about $0.09 \mathrm{fm}$ [12, 13] and the pion and kaon masses are approximately equal to 203 and $520 \mathrm{MeV}$ [11], respectively.

Using a representative ensemble of 150 gauge configurations, the values obtained for the timedependent condensate do indeed have small statistical errors (see table 1). As a function of the flow time $t$, the condensate decreases monotonically, but this trend is practically compensated by the ratio of $G$-factors multiplying the condensate in eq. (3.5). The chiral correction (3.7) thus appears to be small at the quark masses and flow times considered. Moreover, insertion of the lattice spacing 
$a=0.08995(40) \mathrm{fm}$ and the renormalization constant $Z_{P}=0.5800(47)$ of the pseudo-scalar density determined by the PACS-CS collaboration [13-15] yields

$$
\Sigma_{t} \frac{G_{\pi}}{G_{\pi, t}}=[287(2) \mathrm{MeV}]^{3} \quad \text { at } \quad \sqrt{8 t}=0.5 \mathrm{fm}
$$

for the renormalized ratio in the $\overline{\mathrm{MS}}$ scheme at $2 \mathrm{GeV}$, i.e. a value well within the range expected from previous calculations of the condensate $\Sigma$.

\section{Small flow-time expansion}

In all these calculations, no power-divergent terms were encountered nor were there any large contributions that had to be subtracted or extrapolated away. Through the small flow-time expansion, some further insight can be gained into how exactly the power divergences are avoided.

\subsection{General form of the expansion}

Let $\mathcal{O}_{t}(x)$ be a gauge-invariant local field in the continuum theory at flow time $t>0$. Since its footprint decreases proportionally to $\sqrt{8 t}$, the field looks more and more like a strictly local field when $t$ is taken to zero. Eventually, $\mathcal{O}_{t}(x)$ can be represented through an asymptotic expansion [2]

$$
\mathcal{O}_{t}(x) \underset{t \rightarrow 0}{\sim} \sum_{k} c_{k}(t) \phi_{k}(x)
$$

in a series of local fields $\phi_{k}(x)$ at vanishing flow time with time-dependent coefficients $c_{k}(t)$. The expansion holds when inserted in correlation functions at non-zero distances and if all fields are properly renormalized. Clearly, for the expansion to be completely well defined, the chosen renormalization conditions must fix both the normalization and mixing of the fields $\phi_{k}(x)$.

The expansion coefficients $c_{k}(t)$ satisfy a renormalization group equation that determines their asymptotic behaviour

$$
c_{k}(t) \underset{t \rightarrow 0}{\propto} t^{\frac{1}{2}\left(d_{k}-d_{O}\right)} \bar{g}^{v_{k}}\left\{1+\mathrm{O}\left(\bar{g}^{2}\right)\right\}
$$

at small flow times. In this equation, $d_{k}$ and $d_{O}$ are the engineering dimensions of the fields $\phi_{k}(x)$ and $\mathcal{O}_{t}(x)$, the exponents $v_{k}$ are determined by the one-loop coefficients of their anomalous dimensions and $\bar{g}$ denotes the running coupling (in any scheme) at momentum $(8 t)^{-1 / 2}$ [饮. The fields $\phi_{k}(x)$ with the lowest dimension thus dominate in the limit $t \rightarrow 0$, all other terms being suppressed by powers of $t$.

\subsection{Example: expansion of the chiral densities (3.1)}

The asymptotic expressions for the expansion coefficients $c_{k}(t)$ simplify considerably if the renormalization-group-invariant (RGI) normalization convention is adopted for the fields and the quark mass matrix $M$ (the RGI convention is described in sect. 2.2 of ref. [16], for example). In the case of the densities (3.1), the small flow-time expansion then assumes the form

$$
\begin{aligned}
& S_{t}^{r s}(x)=c_{0}(t) M^{r s}+c_{1}(t) \operatorname{tr}\left\{M^{2}\right\} M^{r s}+c_{2}(t)\left(M^{3}\right)^{r s}+c_{3}(t) S^{r s}(x)+\mathrm{O}(t), \\
& P_{t}^{r s}(x)=c_{3}(t) P^{r s}(x)+\mathrm{O}(t), \\
& c_{0}(t)=-\frac{3}{8 \pi^{2} t}\left\{1+\mathrm{O}\left(\bar{g}^{2}\right)\right\}, \quad c_{3}(t)=\left(2 b_{0} \bar{g}^{2}\right)^{-8 / 9}\left\{1+\mathrm{O}\left(\bar{g}^{2}\right)\right\},
\end{aligned}
$$


where $b_{0}$ is the one-loop coefficient of the QCD $\beta$-function. The structure of these expansions is partly dictated by chiral symmetry. Moreover, the asymptotic relation

$$
c_{3}(t)=\frac{G_{\pi, t}}{G_{\pi}}+\mathrm{O}(t)
$$

holds, as can easily be shown by inserting eq. (4.4) in eq. (3.4).

When $t$ is sent to zero, the first term in the expansion (4.3) of the scalar density blows up like $1 / t$ and eventually makes the dominant contribution to the time-dependent condensate $\Sigma_{t}$. Clearly, for the ratio $\Sigma_{t} G_{\pi, t} / G_{\pi}$ to be close to $\Sigma$, the flow time and the quark masses must be such that this singular term is much smaller than $\Sigma_{t}$. Some rough estimates suggest that this condition is indeed satisfied in the numerical experiment reported earlier.

\subsection{A broader perspective}

The small flow-time expansion may also be used to represent any gauge-invariant local field $\phi(x)$ at vanishing flow time through an asymptotic series of local fields at some positive flow time $t$. In the simplest cases, where the fields are uniquely characterized by their dimension and symmetry properties, the representation assumes the form

$$
\phi(x)=c(t) \mathcal{O}_{t}(x)+\mathrm{O}(t)
$$

with a coefficient $c(t)$ that varies at most logarithmically as $t$ goes to zero. Fields where such a representation may conceivably be worth considering include the energy-momentum tensor [17] and the various parts of the effective electro-weak Hamiltonian.

The use of local fields at non-zero flow time is attractive, because their renormalization and $\mathrm{O}(a)$-improvement [3] is very much simpler than the one of the ordinary local fields [19-21]. As already noted, the renormalization is always multiplicative by a power of the quark-field renormalization constant $Z_{\chi}$. The $\mathrm{O}(a)$-improvement, on the other hand, is achieved by including a mass correction

$$
\begin{aligned}
& Z_{\chi} \rightarrow Z_{\chi}\left(1+b_{\chi} a m_{\mathrm{q}, r}+\bar{b}_{\chi} \operatorname{tr} M_{\mathrm{q}}\right), \\
& M_{\mathrm{q}}=\operatorname{diag}\left(m_{\mathrm{q}, u}, m_{\mathrm{q}, d}, \ldots\right): \text { bare quark mass matrix },
\end{aligned}
$$

in the renormalization factor of the quark field with flavour index $r$ and by adding a contact term

$$
\overline{\chi(t, x) \bar{\chi}}(s, y) \rightarrow a^{8} \sum_{v, w} K(t, x ; 0, v)\left\{S(v, w)-a c_{\mathrm{fl}} \delta(v-w)\right\} K(s, y ; 0, w)^{\dagger}
$$

to the quark propagator $S(v, w)$ in the contractions of the quark fields at positive flow times. The kernel $K(t, x ; s, y)$ in eq. (4.10) denotes the smoothing factor determined by the quark flow equation and $c_{\mathrm{fl}}$ is a new improvement coefficient that can be determined non-perturbatively from a combination of pseudo-scalar correlation functions [3]. In particular, $\mathrm{O}(a)$-improvement does not require subtractions of higher-dimensional fields.

For field representations such as (4.7) to work out in practice, one must however be able to accurately determine the coefficients multiplying the time-dependent fields. In perturbation theory, 
the coefficients of the fields of dimension 4 in the expansion of the energy-momentum tensor in the pure gauge theory were recently computed to one-loop order [17]. Non-perturbative calculations of some of these coefficients may also be possible using the Ward identities that derive from the conservation of the energy-momentum tensor in the continuum theory [18]. Another more widely applicable method is to extract the coefficients from the asymptotic relations obtained by inserting the field representation in suitable correlation functions, as in the case of the coefficient (4.6), but whether step-scaling strategies can be used in this context is not clear at present.

Once the coefficients are known, calculations of hadronic matrix elements will, in general, require a "scaling window", where

$$
a \ll \sqrt{8 t} \ll \text { relevant low-energy scales, }
$$

as otherwise the lattice effects and the systematic errors deriving from the neglected terms in the field representation are not guaranteed to be small. In this respect, the computation of the chiral condensate along the lines described in this talk is a somewhat special case, because the contributions of the neglected terms either vanish in the chiral limit or cancel in the product $\Sigma_{t} G_{\pi} / G_{\pi, t}$.

\section{Wilson's renormalization group revisited}

In 1979 Wilson gave a memorable lecture at Cargèse, where he proposed to use the "blockspin renormalization group" for non-perturbative renormalization and the construction of coarse-grid actions in non-Abelian gauge theories [22]. An assumption made at the time was that the expectation values of iteratively blocked Wilson loops have a continuum limit. Wilson was well aware of this, but remarked that a proof of scaling in lattice perturbation theory would be "rather complicated to carry out".

We may now consider replacing the link blocking by the gradient flow and to combine the latter with step scaling [23], which may be regarded as a particular incarnation of the renormalization group. For simplicity, the quark masses are set to zero in the following, but the extension of the discussion to the general case is straightforward and is of some interest, since mass-independent renormalization schemes are inappropriate for the charm and the heavier quarks.

\subsection{Step scaling}

Step scaling is a finite-size scaling method, where the gauge coupling and the renormalizationscale-dependent fields run with the lattice size $L$ (see fig. 3). The precise choice of the normalization conditions for the coupling and fields is only of practical importance in this context. In particular, using the gradient flow, a possible definition of the running coupling is [1, 24-26]

$$
\bar{g}^{2}(L)=\text { constant } \times\left\{t^{2}\left\langle E_{t}\right\rangle\right\}_{\sqrt{8 t}=\frac{1}{3} L} .
$$

As discussed before, the field $E_{t}$ does not require renormalization. Moreover, since the flow time $t$ in eq. (5.1) is scaled proportionally to $L^{2}$, the coupling depends on a single external scale and thus runs with $L$ as it should (the proportionality constant is a parameter of the renormalization scheme, which may in principle be set to any value [25]). 


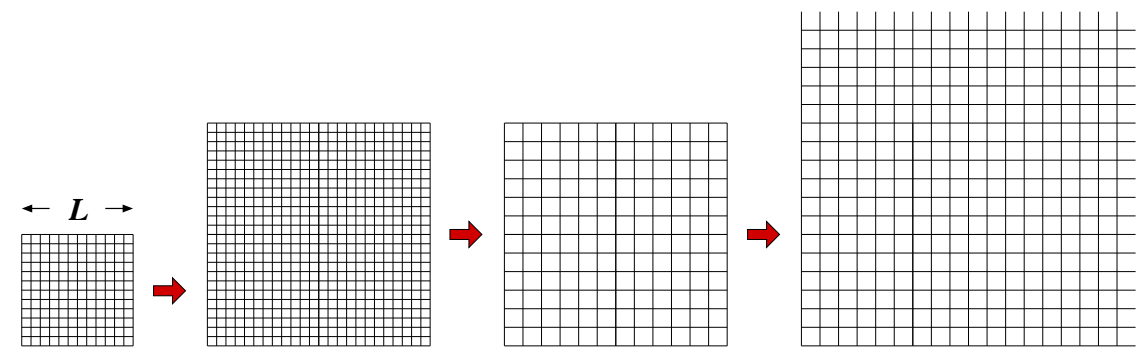

Figure 3: In step-scaling calculations, the scale evolution amounts to changing the lattice size $L$ at fixed lattice spacing $a$ (i.e. fixed bare coupling) by, say, a factor 2 . Then follows a renormalization step, where the running coupling $\bar{g}^{2}(L)$ (and thus $L$ in physical units) is held fixed, while $a$ is increased by a factor 2. After that the process continues with another scale evolution step, and so on.

The coupling (5.1) has some good technical properties that should allow step-scaling studies to be performed with unprecedented precision. In particular, its computation in numerical lattice QCD does not require any fits or extrapolations to be performed, nor does its variance increase significantly towards the large-volume regime of the theory [26], where contact is made with the non-perturbative low-energy scales of the theory.

\subsection{Construction of improved actions}

Step scaling is usually combined with an extrapolation to the continuum limit of the so-called step-scaling functions [23]. The calculation then effectively solves the non-perturbative renormalization problem in the continuum theory. When constructing improved lattice actions, the goal is a different one, namely to reduce the lattice effects as much as possible after having properly taking into account the parameter and field renormalization.

For practical reasons, improved lattice theories may not be too complicated. Their construction must therefore start from a suitable ansatz for the action and the improved fields with a reasonably small number of parameters. The tuning of the parameters of the improved theory then proceeds by matching lattices with different spacings as in fig. 4 [22].

To be able to do this, a sufficiently large set of accurately computable renormalized quantities is needed. So far this requirement was difficult to meet, but there is now new hope that progress can be made using observables based on the Yang-Mills gradient flow and the associated quark flow. The expectation values of the fields

$$
\begin{aligned}
& \operatorname{tr}\left\{G_{\mu v} G_{\mu v}\right\}, \quad \bar{\chi} \chi, \quad \bar{\chi} \sigma_{\mu v} G_{\mu v} \chi \\
& (\bar{\chi} \Gamma \chi)(\bar{\chi} \Gamma \chi), \quad \Gamma \in\left\{1, \gamma_{5}, \gamma_{\mu}, \gamma_{\mu} \gamma_{5}, \sigma_{\mu v}\right\}
\end{aligned}
$$

are simple examples of such quantities, and there is obviously a wide range of further observables, local and non-local ones, that may serve the purpose. Moreover, all of them can be considered at several flow times $t$. Being able to probe the theory in many different channels is important in order exclude a situation, where the parameter tuning removes some small lattice effects in one channel but produces large effects elsewhere. 


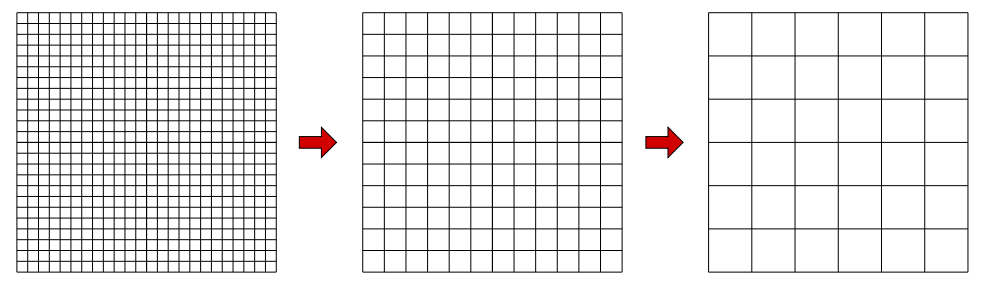

Figure 4: At fixed coupling $\bar{g}^{2}(L)$, renormalized quantities are independent of the lattice spacing up to lattice effects. Improved actions and fields can therefore be tuned by simulating sequences of matched lattices and by minimizing the differences in the measured values of a suitable set of such quantities.

\section{Concluding remarks}

Some of the ideas discussed in this talk clearly need to be further developed, through both analytical and numerical studies, before their viability can be assessed. The Yang-Mills gradient flow and its extension to the quark fields however stand on solid theoretical ground and there are already a few important applications of the flow, which are known to work out in practice.

The fact that the power-divergent mixing of the scalar quark density with the unit field can be bypassed by going to non-zero flow time is intriguing and suggests that the same may perhaps be possible in other cases of field mixing as well. Computations of electro-weak transition matrix elements, for example, might profit from such a change of strategy, but the application of the YangMills gradient flow in this context probably belongs to the more distant future.

\section{Acknowledgments}

In the course of the preparation of this talk, I have had many interesting and stimulating discussions on the subject with Agostino Patella, Roberto Petronzio and Hiroshi Suzuki. I also wish to thank Stefan Schaefer and Peter Weisz for a pleasant and fruitful collaboration in the past few years. All numerical simulations reported here were performed on a dedicated PC cluster at CERN. I am grateful to the CERN management for providing the required funds and to the CERN IT Department for technical support.

\section{References}

[1] M. Lüscher, Properties and uses of the Wilson flow in lattice QCD, JHEP 08 (2010) 071.

[2] M. Lüscher, P. Weisz, Perturbative analysis of the gradient flow in non-Abelian gauge theories, JHEP 02 (2011) 051.

[3] M. Lüscher, Chiral symmetry and the Yang-Mills gradient flow, JHEP 04 (2013) 123.

[4] M. F. Atiyah, R. Bott, The Yang-Mills equations over Riemann surfaces, Phil. Trans. R. Soc. Lond. A 308 (1982) 523.

[5] C. Morningstar, M. Peardon, Analytic smearing of SU(3) link variables in lattice QCD, Phys. Rev. D69 (2004) 054501.

[6] S. Güsken, A study of smearing techniques for hadron correlation functions, Nucl. Phys. (Proc. Suppl.) B17 (1990) 361. 
[7] C. Alexandrou, F. Jegerlehner, S. Güsken, K. Schilling, R. Sommer, B meson properties from lattice QCD, Phys. Lett. B256 (1991) 60.

[8] J. Zinn-Justin, D. Zwanziger, Ward identities for the stochastic quantization of gauge fields, Nucl. Phys. B295 [FS21] (1988) 297.

[9] J. Gasser, H. Leutwyler, Chiral perturbation theory to one loop, Ann. Phys. 158 (1984) 142.

[10] M. Lüscher, S. Schaefer, Lattice QCD without topology barriers, JHEP 07 (2011) 036.

[11] M. Lüscher, S. Schaefer, Lattice QCD with open boundary conditions and twisted-mass reweighting, Comput. Phys. Commun. 184 (2013) 519.

[12] S. Aoki et al. (PACS-CS collab.), 2+1 flavor lattice QCD toward the physical point, Phys. Rev. D79 (2009) 034503.

[13] S. Aoki et al. (PACS-CS collab.), Physical point simulation in 2+1 flavor lattice QCD, Phys. Rev. D81 (2010) 074503.

[14] S. Aoki et al. (PACS-CS collab.), Non-perturbative renormalization of quark mass in $N_{f}=2+1 Q C D$ with the Schrödinger functional scheme, JHEP 08 (2010) 101.

[15] Y. Taniguchi, private communication.

[16] P. Weisz, Renormalization and lattice effects, in: Modern perspectives in lattice QCD (Les Houches 2009), eds. L. Lellouch et al. (Oxford University Press, Oxford, 2011) [arXiv:1004.3462].

[17] H. Suzuki, Energy-momentum tensor from the Yang-Mills gradient flow, Prog. Theor. Exp. Phys. 2013 083B03.

[18] L. Del Debbio, A. Patella, A. Rago, Space-time symmetries and the Yang-Mills gradient flow, arXiv:1306.1173.

[19] B. Sheikholeslami, R. Wohlert, Improved continuum limit lattice action for QCD with Wilson fermions, Nucl. Phys. B259 (1985) 572.

[20] M. Lüscher, S. Sint, R. Sommer, P. Weisz, Chiral symmetry and $O(a)$ improvement in lattice $Q C D$, Nucl. Phys. B478 (1996) 365.

[21] T. Bhattacharya, R. Gupta, W. Lee, S. R. Sharpe, J. M. S. Wu, Improved bilinears in lattice QCD with nondegenerate quarks, Phys. Rev. D73 (2006) 034504.

[22] K. G. Wilson, Monte-Carlo calculations for the lattice gauge theory, in: Recent developments in gauge theories (Cargèse 1979), eds. G. 't Hooft et al. (Plenum Press, New York, 1980).

[23] M. Lüscher, P. Weisz, U. Wolff, A numerical method to compute the running coupling in asymptotically free theories, Nucl. Phys. $\mathbf{B 3 5 9}$ (1991) 221.

[24] Z. Fodor, K. Holland, J. Kuti, D. Nogradi, C. H. Wong, The Yang-Mills gradient flow in finite volume, JHEP 11 (2012) 007; The gradient flow running coupling scheme, PoS (Lattice 2012) 050.

[25] P. Fritzsch, A. Ramos, The gradient flow coupling in the Schrödinger functional, arXiv:1301.4388; Studying the gradient flow coupling in the Schrödinger functional, arXiv:1308.4559.

[26] A. Ramos, The gradient flow in a twisted box, arXiv:1308.4558. 